\title{
The sPHENIX TPC Project
}

\author{
Klaus Dehmelt* \\ Department of Physics and Astronomy \\ Stony Brook University \\ E-mail: klaus.dehmelt@stonybrook.edu
}

\begin{abstract}
A state-of-the-art detector, called sPHENIX, is being planned at RHIC for measuring jets, jet correlations and upsilons to study the microscopic nature of the Quark-Gluon Plasma. Precision vertexing and tracking, as well as calorimetry, will be provided over full azimuth and pseudorapidities of $|\eta|<1.1$. The data will be continuously recorded making use of the $15 \mathrm{kHz}$ RHIC collision rate in the region of interest.

A high resolution and low mass tracking system for reconstructing the three upsilon states is needed. The tracking system comprises silicon pixel and strip detectors and a compact TPC. The TPC at the present planning stage will be using quadruple-GEM detectors as a readout, following the path of the ALICE-TPC upgrade, with a modified Neon-based gas mixture. The main tasks of this configuration are the achievement of sufficient momentum resolution and combating ion back-flow.
\end{abstract}

The motivation and the design of the technology choices will be presented along with the present status of the project. Furthermore, alternative readout structures, like hybrids of MicroMegas and GEMs will be discussed.

5th International Conference on Micro-Pattern Gas Detectors (MPGD2017)

22-26 May, 2017

Philadelphia, USA

\footnotetext{
*Speaker.

${ }^{\dagger}$ For the sPHENIX Collaboration.
} 


\begin{tabular}{|c|c|}
\hline Physics Goals & Detector Requirements \\
\hline Fragmentation functions & Excellent momentum resolution: $\mathrm{dp} / \mathrm{p} \sim 0.2 \%$ for $\mathrm{p}>40 \mathrm{GeV} / \mathrm{c}$ \\
Jet substructure & Excellent track pattern recognition \\
Distinguish $\Upsilon$-states & Mass resolution $\sigma_{m}<100 \mathrm{MeV} / \mathrm{c}^{2}$ \\
Heavy flavor jet tagging & Precise DCA resolution $\sigma_{D C A}<100 \mu \mathrm{m}$ \\
High statistics Au+Au $200 \mathrm{GeV}$ & Handle multiplicity and full RHIC luminosity \\
\hline
\end{tabular}

Table 1: Physics goals and detector requirements for sPHENIX.

\section{Introduction}

The sPHENIX experiment at the Relativistic Heavy Ion Collider (RHIC) [1] will consist of a high-resolution and low mass tracking system embedded in a sandwiched electromagnetic and hadronic calorimetric system as well as a compact superconducting magnet. It aims for a high data acquisition rate of $15 \mathrm{kHz}$ in the region of interest (vertex) and needs to handle an estimated peak collision rate of $200 \mathrm{kHz}$.

The physics goals as well as the detector requirements are summarized in table 1 . The goals will be accomplished by a tracking system that comprises a three-layer Si-pixel detector (MAPS), a four-layer Si-strip detector (Intermediate tracker) and a compact time projection chamber (TPC). The TPC requires a continuous readout system and at the same time small space charge distortions. The dimension of the barrel solenoid (former BaBar magnet with $\mathrm{B}=1.4 \mathrm{~T}$ ) dictates the size of the TPC, which will have a radius of $20 \mathrm{~cm}<\mathrm{R}<75 \mathrm{~cm}$ and a length of $211 \mathrm{~cm}$, and full azimuthal coverage. This results in a pseudo-rapidity range of $|\eta|<1.1$. Due to the requirement of

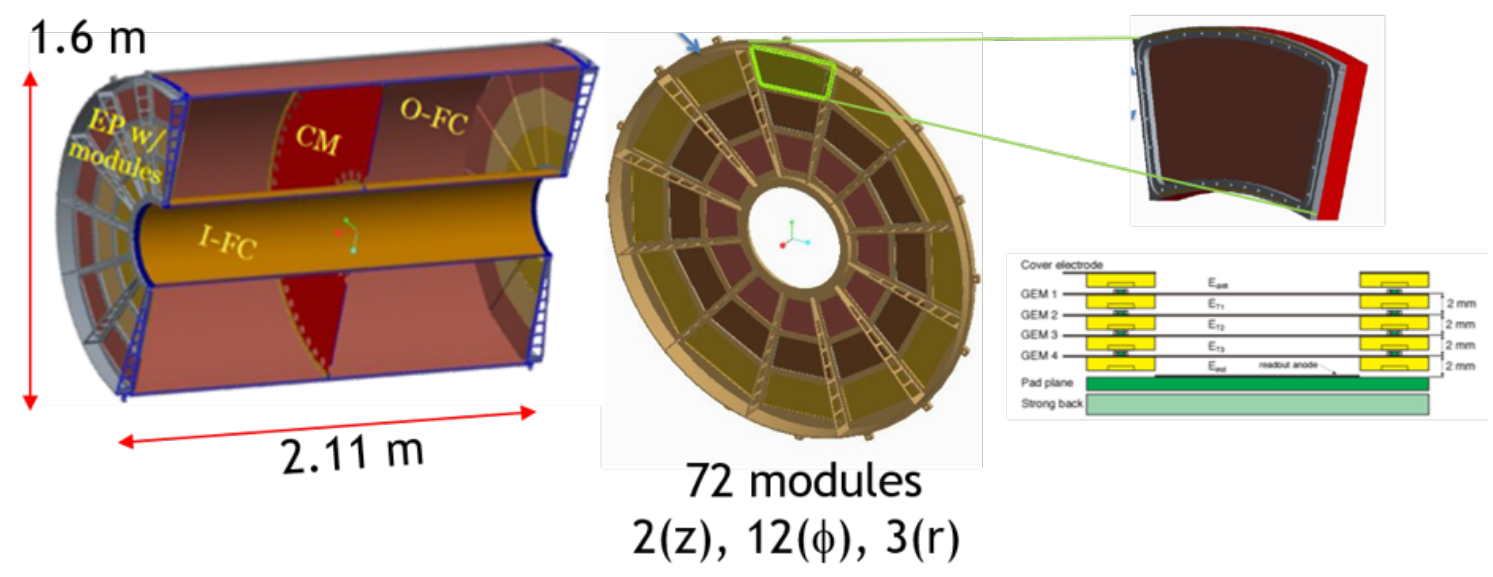

Figure 1: The sPHENIX TPC with a close-up look onto the end-plate and readout modules.

continuous and high-rate data acquisition a traditional gating option for eliminating ion-back flow (IBF) renders unusable. However, to reduce IBF to a manageable level the readout has to consist of micro-pattern gas detectors (MPGD) which provide intrinsic IBF blocking features. Gas Electron Multiplier (GEM [2]) and Micro-mesh gas (MicroMegas[3]) detectors are the technologies most used nowadays. The upgrade project of the ALICE-TPC [4] is based on a novel TPC concept that 
the ALICE collaboration has developed. Many of these developments will be taken as the choice and will be applied, amongst others, to make the compact TPC for use in sPHENIX feasible.

The sPHENIX TPC is a cylindrical detector that is divided in the center (around the interaction point) by a central membrane, with an inner and outer field-cage and a closure in form of end-plates on either sides that accommodate the readout modules (see Fig. 1). The details of the construction will be discussed in the following sections.

\section{Field Cage}

The field cage of the sPHENIX TPC is a hybrid between two TPCs, either presently operated or under investigation: STAR [5] and a large prototype of the ILD-TPC [6]. Owing to the fact that the sPHENIX TPC is rather compact compared to other TPCs it needs to use up all possible space to provide enough lever arm for tracks recorded. Consequently, the field cage wall needs to be as thin as possible though provide maximal mechanical and electrical stability. It will provide both, the field shaping element of the drift volume as well as the gas vessel.

The field cage has been studied regarding mechanical and electrostatic tolerances. Simulations have been performed and the following imperfections were implemented: • Module tilt, $\bullet$ Membrane tilt, $\bullet$ Shifted field cage, $\bullet$ Rotated field cage, $\bullet$ Resistor tolerance. In the following electric field

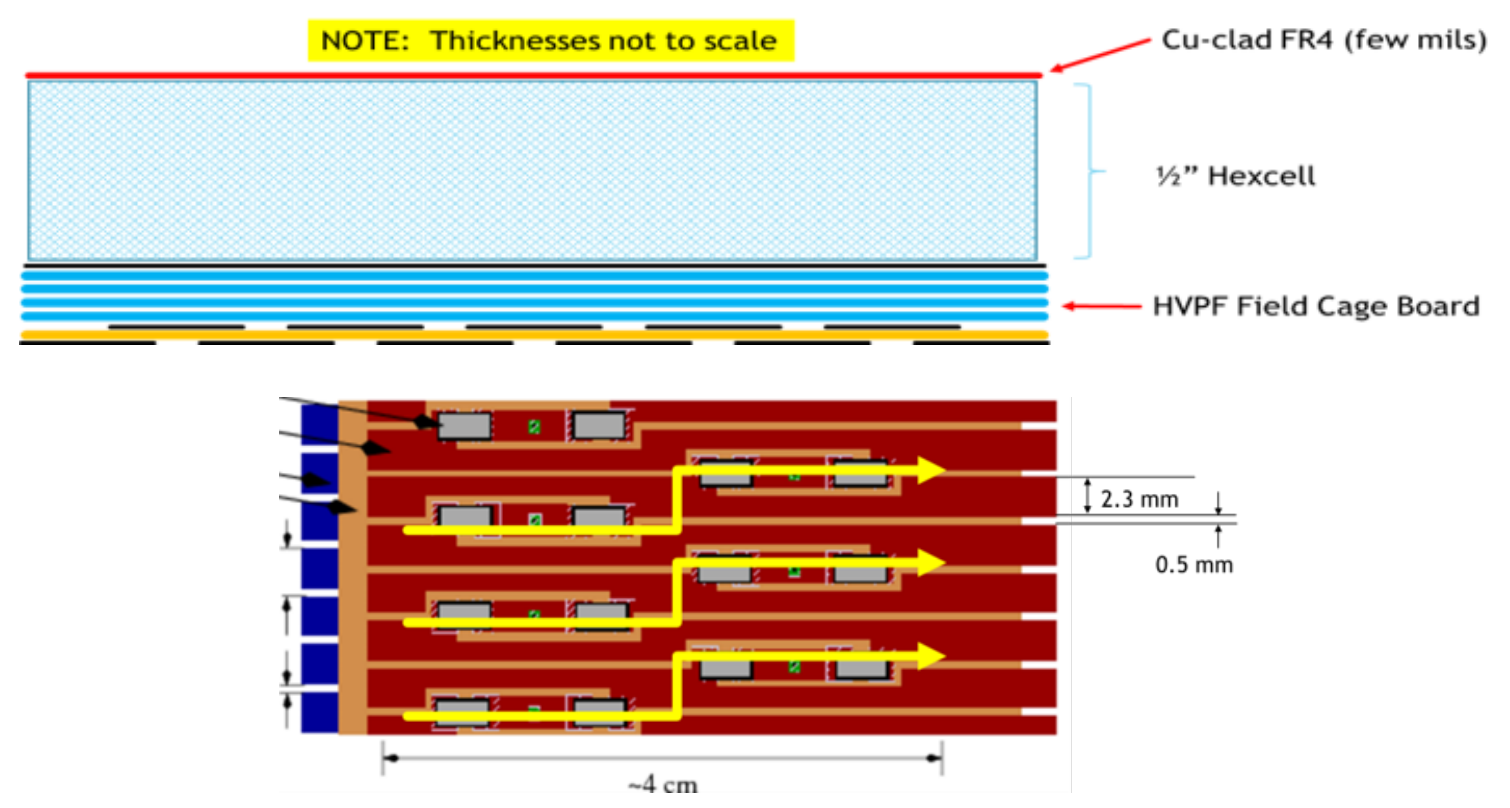

Figure 2: Upper: Components of the field cage wall for the sPHENIX TPC. The high voltage polymer foil (HVPF) guarantees the high-voltage stability. Lower: Close-up look at the field-strip foil.

distributions for the various cases were calculated with a finite element method (FEM, ANSYS) within the field cage. Based on these field maps the distortions of the drift path of electrons at various radii in the field cage for several magnetic fields were simulated based on the Garfield simulation program [7]. As an example Fig. 3 shows that the distortions are acceptable at magnetic fields planned in SPHENIX. 

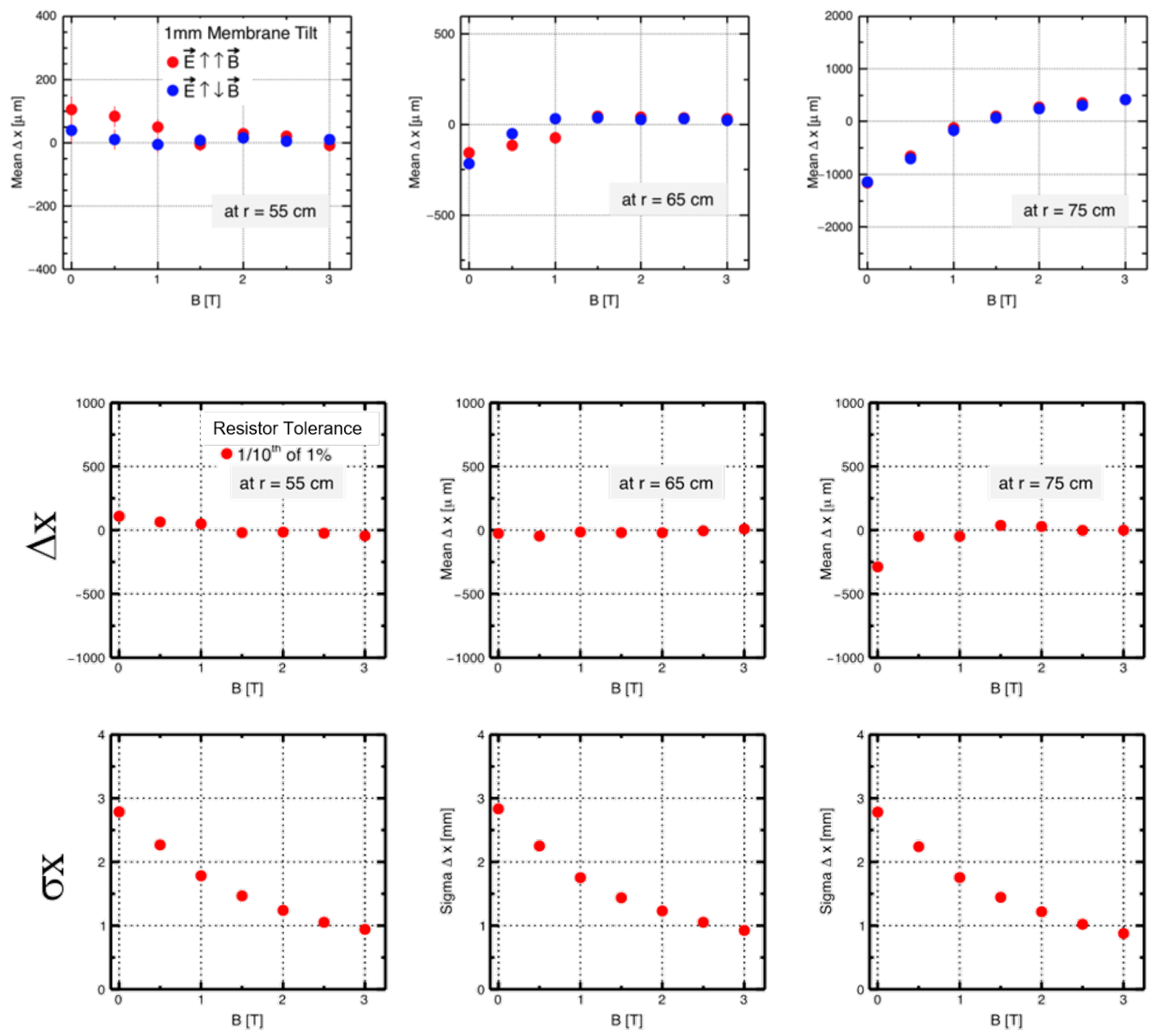

Figure 3: Upper row: Mean deviation and their sigma of electron paths based on a membrane tilt of $1 \mathrm{~mm}$ w.r.t. nominal position. Lower two rows: Random variation of resistor values by $1 \%$ of the nominal value.

\section{Readout Modules}

The module design for the sPHENIX TPC amplifying and reading out the signals coming from the drift volume is following close to the design that was developed for the ALICE-TPC upgrade. The readout is subdivided into 72 modules. This accounts for the two end-plates having each three radial divisions and twelve azimuthal divisions per radius. The present concept is going with a quadruple-GEM system for each module (Fig. 1, right). Each module will consist of a stack of four GEMs with different pitch and misalignment relative to each other, see Fig. 4. The pad plane will consist of zig-zag shaped pads of dimensions $\sim 2 \mathrm{~mm}$ in $\mathrm{r}-\phi$ and $\sim 10 \mathrm{~mm}$ in r, see Fig. 5 . The zig-zag shape has been chosen because it promises the increased probability of charge sharing though having rather large pad size: the pad pitch and zig-zag period has been chosen such that it matches the size of the charge cloud $(\sim 500 \mu \mathrm{m})$. The zig-zag design also ensures that the charge sharing is directly proportional to the hit position. 


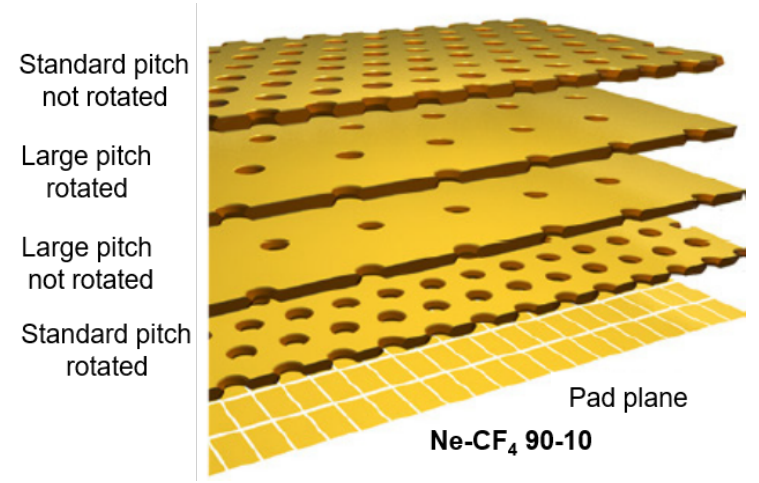

Figure 4: Stack of four GEMs, with different pitch and hole pattern (from [8]). The counting gas foreseen is $\mathrm{Ne}_{-} \mathrm{CF}_{4}$ with a ratio of $90-10$.

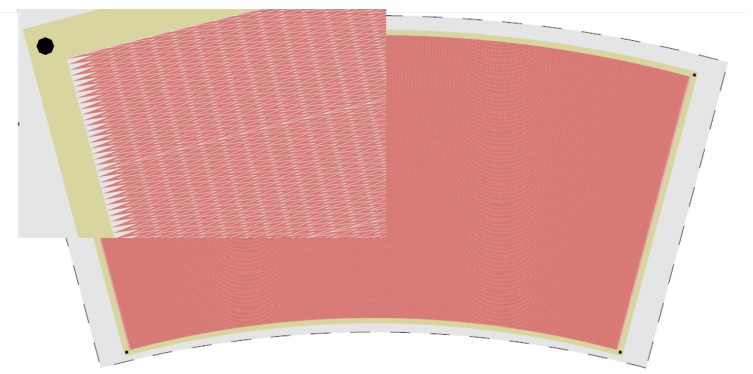

Figure 5: Annular shaped pad plane with zig-zag shaped pads. The inset shows details of the pad design.

\section{Space Charge}

In a heavy-ion collision environment such as at the RHIC a high track density is accounted for which in turn creates a high density of ionization products along the tracks. Electrons as the ionization products is required for detecting the tracks, however, each electron also has a positive ion partner. This ion is drifting with much lower speed to the cathode and consequently spends considerable time in the drift volume. Because of large abundance the ions produce a space charge effect which will distort the electric field homogeneity. As a result, uncertainties about the electrons drift arise which will consequently deteriorate the track detection. On top of the ions produced in the drift volume, an even larger number of ions is produced at the avalanche stage (for the sPHENIX TPC $2000 \times$ per primary charge) and is called ion-back flow (IBF). Traditionally, IBF is suppressed by operating a gating grid upon receipt of a trigger, holding it open for a time sufficient to collect electrons with the largest drift time (i. e. those originating near the central membrane), and then closing it for a time period sufficient to block all avalanche-induced positive ions from entering the main TPC gas volume. Because of the "off-time" for responding to positive ions, traditional TPCs cannot be operated with a continuous readout as required for the sPHENIX program. One of the utmost priorities for the sPHENIX TPC is to provide techniques that combat IBF. Such techniques are the following but not limited to: 1) Make the ions fast through mass, 2) Choose the largest drift field possible, 3) Optimize amplification device's operating point, 4) Update design of field cage informed by current experience. 


\subsection{Optimization GEMs Operating Point}

A new concept in limiting IBF has been pioneered by the ALICE collaboration and is expected to be brought on-line by them prior to first data-taking with sPHENIX. With the advent of MPGD technology a breakthrough is possible in IBF handling. As indicated in Fig. 4, the avalanche stage of a gas detector can be made using a stack of Gas-Electron Multiplier (GEM) foils. Each foil contributes a small fraction of the total gain, which is achieved only when avalanching through the full stack. However, through clever manipulation of the electric fields between GEM foils ("transfer" fields) one can generate a condition whereby only a very small fraction of the positive ions are able to drift back into the main detector volume. In this way, the detector can be kept fully live at all times.

\subsection{Update Design Field Cage}

Another effect can be made use of to further reduce space charge effects: as positive ions attract electrons and thereby distort their trajectories toward the "middle" radius of the TPC. Space charge distortions maximize at both the inner and outer field cages where the space charge density has a discontinuity. A full calculation of space charge distortions for sPHENIX is shown in Fig. 6. The blue curve indicates a calculation for a TPC spanning the radial range $30-80 \mathrm{~cm}$. The maximum distortion is $2 \mathrm{~cm}$ found exactly at the inner radius. Notice, however, the red curve for a TPC spanning $20-80 \mathrm{~cm}$. At the lowest radius, the distortion is indeed severe $(3 \mathrm{~cm}, 50 \%$ worse than before), however the distortion of the track at $30 \mathrm{~cm}$ is drastically reduced to only $3 \mathrm{~mm}$. Thus, by modifying our TPC design from the originally-proposed version $(30-80 \mathrm{~cm})$ to a new version that spans $(20-80 \mathrm{~cm})$, can can easily reduce space charge to under $1 \mathrm{~cm}$.

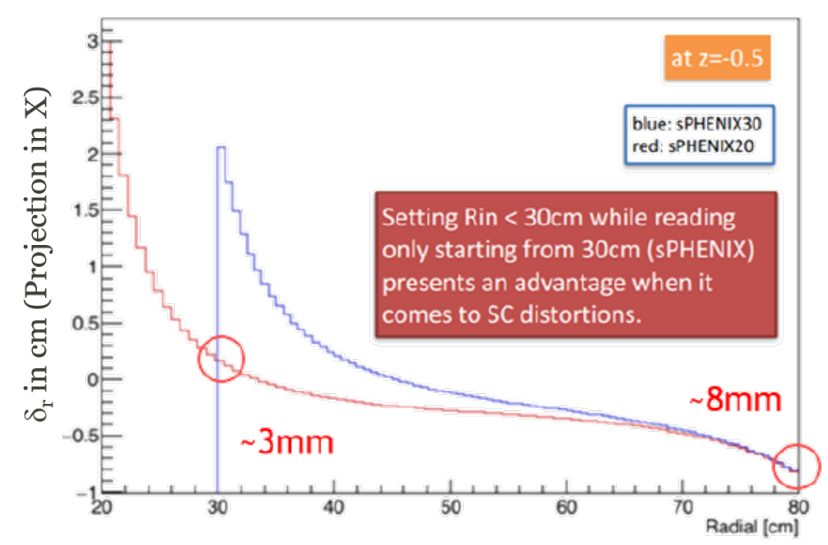

Figure 6: Electron paths are primarily influenced by the charge density closest to the electron. Necessarily, the greatest deflections from the ideal trajectory are found closest to the field cage. By moving the field cage entrance window from $30 \mathrm{~cm}$ to $20 \mathrm{~cm}$, the deflection due to IBF can be reduced to reasonably manageable levels. 


\subsection{Passive Mesh}

There is still significantly more that can be done to reduce IBF. Such a reduction would allow to, for example, change the operation point of the GEM-stack to regain much of the lost resolution. The well understood degradation in energy resolution with decreasing IBF comes from fluctuations at low gain the the first GEM. Indeed, statistical distributions enforce this tendency, for example Poisson distributions have the variable equal to the mean. However, an avalanche is different. At the very least the primary electron in the avalanche will be present at small gain $\sim 1$. For this reason, an avalanche stage with full transparency and no gain introduces no fluctuations. If such a structure were placed with asymmetric entrance and exist field, it is natural to assume that the electric fields would dictate high transparency and low IBF. Full GARFIELD simulations indicate that this configuration should be viable. Many different mesh geometries have been modeled by sPHENIX, one of which is summarized in Figures 7 and 8 . Both the electron transmission (forward
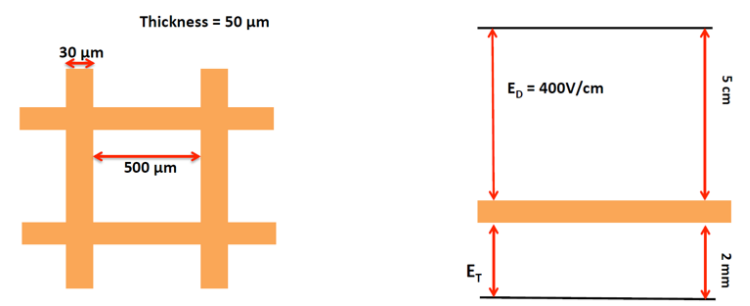

Figure 7: Full GARFIELD simulations including magnetic field in the idealized mesh shape shown here, square holes photographically etched into flat metal.

direction) and the ion blocking (backward direction) have been measured using GARFIELD in our operating gas and as a function of magnetic field in the TPC. Clearly, for quite reasonable ratios of drift and transfer fields, one can achieve nearly $100 \%$ electron transmission while blocking about $80 \%$ of the positive ions. This would, in principle allow for much more favorable operating points with very low IBF and good energy resolution. Future R\&D will confirm these findings.
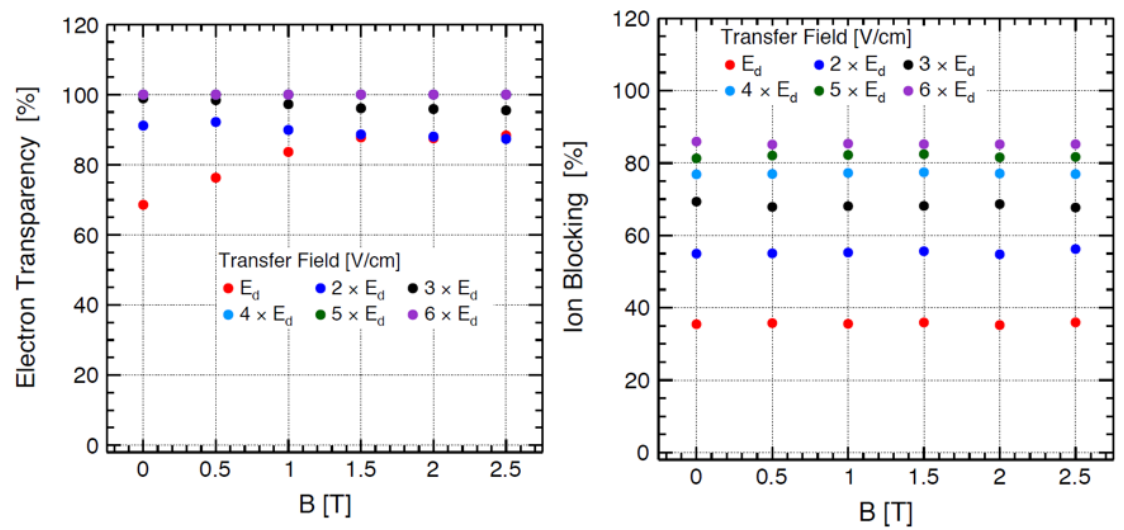

Figure 8: GARFIELD results indicate that for reasonable ratios of $\frac{E_{\text {exit }}}{E_{\text {entrance }}}$ near perfect electron transmission can be achieved while blocking $70-80 \%$ of the ions produced in the avalanche stage. 


\subsection{GEM Amplification and IBF Studies}

A realistic set of studies toward the GEM amplification and IBF has been performed with GARFIELD. Since the modeling of the quad-GEM arrangement with its different pitches and hole arrangements would be rather tedious the full amplification stage was divided in various steps. Simple transformations have been applied to keep the simulation realistic, for details, see Fig. 9.

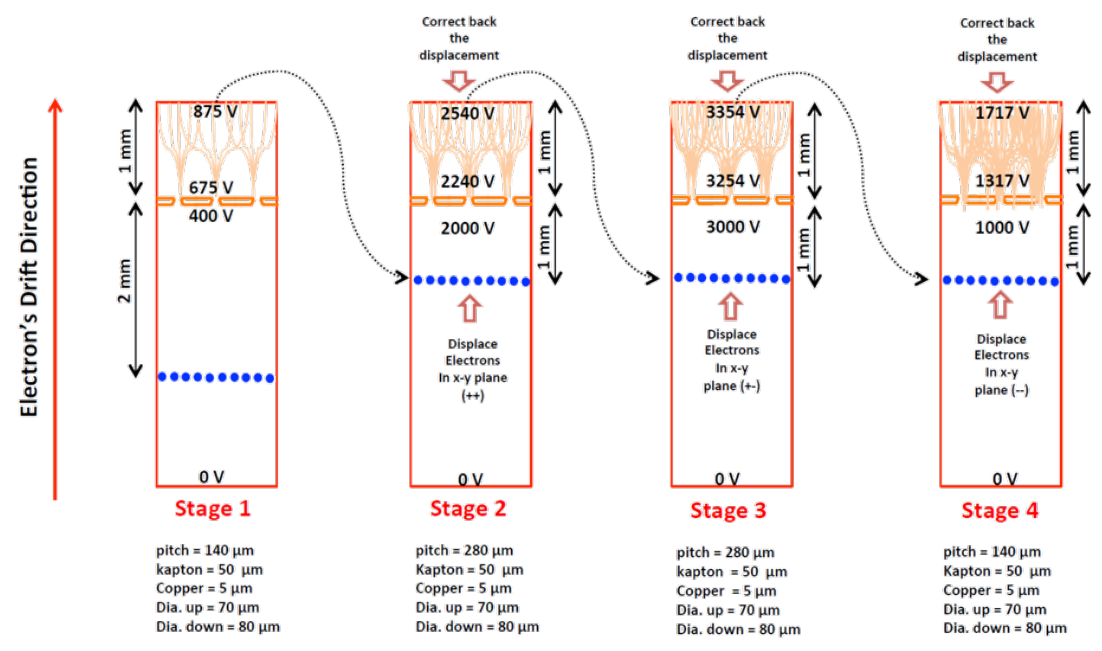

Figure 9: Full quad-GEM simulation with GARFIELD and ANSYS in four separate simulation steps. Each step consists of a single GEM simulation and its outcome is transformed into the coordinate system of the following step.

The outcome for such a simulation is indicated in Fig. 10. It shows the electron losses in stage 3 respectively stage 4 of the quad-GEM stack, as well as the charge cloud that is transferred through these stages.

\section{Readout Electronics}

sPHENIX benefits from the developments in ALICE for their own TPC upgrade. In many ways, the sPHENIX TPC is based upon theirs, in particular the front-end electronics.

The Front End Card (FEC) consists of SAMPA chips [9] which amplify and shape the analog signals and digitize them. The data processing unit (DSP) is also on the chip. This formats the digital data into a data packet (it also performs baseline suppression, i.e., zero-suppression of the raw data).

The sPHENIX FEE cards will each carry 8 SAMPA chips and thereby readout 256 channels on each FEE. Going outward in radius, the sPHENIX modules carry 5, 8, and 12 FEE cards respectively. This results in 153,600 active channels for the entire TPC system. Each sector of 25 FEE cards is serviced by a single PCI-express-based FPGA card, Data Aggregation Module (DAM), which is hosted on a server, Event Buffering and Data Compressor (EBDC). The DAM is responsible for event alignment and clustering. Furthermore, present calculations indicate that we can create false event boundaries from our continuous readout by copying ambiguous data into both triggered events. Then the result sub-event is compressed on EBDC and send to the sPHENIX event builder via Ethernet. 

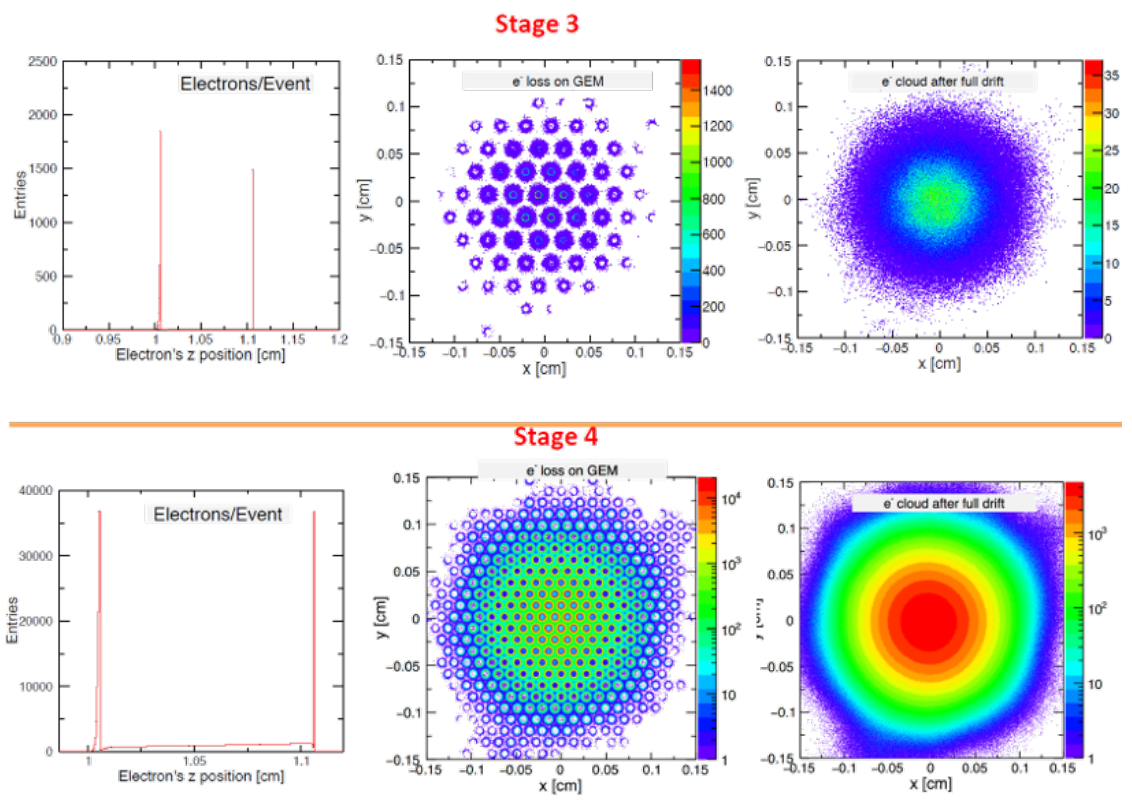

Figure 10: Result of the simulation as indicated in Fig. 9 for Step 3 and Step 4. The three plots indicate from left to right respectively, the electrons' position along the drift-/transfer-direction $\mathrm{z}$, the loss of electrons on the GEM, and the electron cloud after drift.

\section{Summary}

A compact TPC for the sPHENIX experiment was described. The development and construction benefits from existing TPCs as well as from upgrade/new projects of TPCs. An aggressive schedule for the TPC completion is underway. The mechanical design is well defined and a version 1 prototype will already contain a full size field cage. Operating parameters have to be determined, following existing applications. Simulations are promising and will be followed by experimental verification. The IBF as main issue needs to be verified and to be solved. In summary, the sPHENIX TPC is well on track, however, dependent on future developments and findings, possibly some compromises have to be made. Not mentioned int this write-up is that alternative IBF solutions, i.e., alternative gain structures are being looked into.

\section{References}

[1] M. Connors, Nuclear Physics A 967 (2017), p. 548-551

[2] F. Sauli, Nucl. Instr. and Meth. A, 386 (1997), p. 531

[3] Y. Giomataris, Ph. Rebourgeard, J.P. Robert, G. Charpak, Nucl. Instr. and Meth. A, 376 (1996), p. 29

[4] J. Alme et al., Nucl. Instr. Meth. A, 622 (2010), p. 316-367

[5] H. Wieman et al., IEEE TNS, VOL. 44, NO. 3, JUNE 1997

[6] T. Behnke et al., arXiv:1306.6329 [physics.ins-det]

[7] R. Veenhof, Nucl. Instr. Meth. A, 419, (1998), p. 726-730

[8] The ALICE Collaboration, CERN-LHCC-2015-002 / ALICE-TDR-016-ADD-1

[9] S. Barboza et al., JINST, 11 (02) (2016), p. C02088 